\title{
A Masked-Watershed algorithm for segmentation of Brain tumour MR Images
}

\author{
C. Latha \\ Department of Computer Application, \\ Madurai Kamaraj University
}

\author{
K. Perumal, PhD \\ Department of Computer Application, \\ Madurai Kamaraj University
}

\begin{abstract}
Medical imaging creates use of the technology to noninvasively make known the interior structure of the human body. By way of medical imaging modalities patient's life be able to be improved throughout an accurate and rapid remedial without any side effects. The most important reason of this paper is to increased methodology that may accurately classify a tumor growth from abnormal tissues. This examination work has distinguished region growing segmentation Technique and implement and reflect. From our validation test comes about, this examination work uncovered that this model neglects to create useful groups order force effect poor tumor characterization precision. The images used for tumor segmentation are obtained from MRI modality. During this research, we've developed an image segmentation technique based on catchments basins and ridge lines to segment the brain image accurately. This technique relies on interest techniques for extracting tumor portion of the images in the watershed segmentation. Direct application of watershed leads to over-segmentation because of the presence of noise and different irregularities accepted in digital images. Thus to avoid this, we've carried out some preprocessing to eliminate noise present within the MRI images through acquisition stage. Then mask the watershed segmentation. After preprocessing step, we have a tendency to calculate the morphological operation of the input images. This exists one of the real issues to predict the tumor design and to address this issue; the masked watershed Segmentation Technique is proposed and resolute overall Sensitivity, Specificity, and Accuracy.
\end{abstract}

\section{Keywords}

MRI, Brain tumor, morphological operation, Watershed segmentation and Masked-Watershed Segmentation.

\section{INTRODUCTION}

The brain tumor is one in all the foremost necessary causes for the increase in mortality with kids and adults. A tumor is a collection of tissue with the purpose of developing out of control of the normal forces that regulates development in 1993 itself. The complex brain tumors can be divided into two specific categories.

Depending on their origin, growth sample, and malignancy. Primary brain tumors are real tumors that acquire position from the cells within the brain or from the layer of the brain. A secondary or metastatic brain tumor happens once cancer cells spread to the brain from primary cancer in another part of the body.

Most Researches in developed countries show that the amounts of individuals who develop brain tumors and die from them contain greater than earlier than possibly as to a great extent as 300 over past three decades. The TumorSpecific Statistics are represented as below
Meningiomas represent $36.4 \%$ of all primary brain tumors, creating them the foremost common primary brain tumor. There'll be an estimated 24,880 new cases in 2016.Gliomas, a broad expression which includes all tumors take place from the supportive tissue of the brain, correspond to $27 \%$ of the entire brain tumors and $80 \%$ of the entire malignant tumors. Glioblastomas represent $15.1 \%$ of the entire primary brain tumors and $55.1 \%$ of the entire gliomas. Glioblastoma has the maximum number of cases of all malignant tumors, with an estimated 12,120 original cases predicted in 2016. Astrocytomas, as well as glioblastoma, represent just about $75 \%$ of all gliomas. Nerve sheath tumors/acoustic neuromas characterize about $8 \%$ of all primary brain tumors. Pituitary tumors correspond to $15.5 \%$ of the entire primary brain tumors. There resolve to be an estimated 11,700 new cases of pituitary tumors in 2016. Lymphomas stand for $2 \%$ of all primary brain tumors. Oligodendrogliomas stand for nearly $2 \%$ of all primary brain tumors. Medulloblastomas/primitive tumors represent $1 \%$ of all primary brain tumors. The greater part of primary tumors $(36.4 \%)$ is placed within the meninges. Possible usage for brain tumors differs fairly a bit stand on the size and position of the tumor in the brain, at the same time as the person's overall health and risk factors. Usually, on the other hand, the initial step is the removal of the tumor, generally through some type of surgical procedure. After that, chemotherapy, radiation therapy or a mixture of the two treatments is placed to eradicate cancer from neighboring tissue and hopefully prevent its repetition.

This paper is organized as follows: Section II consist of a literature survey of the brain tumor. Sections III Problem Definition. Sections IV discusses Proposed Methodology used. Sections V shows results analysis and validation. Section VI shows conclusion. Section VII presents references.

\section{LITERATURE SURVEY}

Zhang et al. (2012) [1] has established the adaptive marker extraction-based watershed segmentation be used to overcome the over-segmentation problem. It likewise analyzes the disadvantages of the classical watershed segmentation and near a marked extraction support on adaptive color image segmentation algorithm to get better-watershed segmentation, the conventional markers meant for the lack of extraction methods, a lot of regard as the minimum characteristics of properties, and set the adaptive threshold.

Rah man et al. (2013) [2] has discussed object as well as in an image is one in all the foremost significant challenges in the image processing. Image segmentation is applied to split alike particles, which assist in computing the estimated total number of particles. Threshold technique is attractive for counting objects within an image. It makes use of the markercontrolled watershed segmentation all along with threshold technique make available a suitable result. The shortcoming of 
the method obtains bright objects as an image and dark objects are considered as a background when the particles are of different tones of colors

Fu et al. (2012) [3] Two-step marker-controlled watershed image segmentation method which is in high- speed, in the CIELAB color space to determine the over-segmentation problem, which saves a set of execution time. The watershed excellent pixel segmentation technique creates oversegmented regions powerfully which stay well to the real object boundaries. This process is faster than various other segmentation algorithms and very suitable for real-time applications.

Li et al. (2012) [4] has suggested the latest approach to reducing over-segmentation by means of both pre and post processing for watershed segmentation. A new watershed segmentation method that merges pre-processing of the image and post-processing of image objects to produce the final segmentation results. In the early stage of the watershed transform, this not only generates a gradient image from the original image. It also introduces the texture gradient. The texture gradient can be obtained using a gray-level cooccurrence matrix.

Richhariya et al. (2013) [5] has considered the merging morphological watershed result with improved edge detection end result take degraded images. In a post-processing step, to each one of the segmented regions achieve, color histogram algorithm is applied, improving the overall presentation of the watershed algorithm. Image deblurring can be finished with the 2-D Gaussian function.

Acharjya et al. (2013) [6] has an obtainable novel approach of Watershed Algorithm by means of Distance Transform is be relevant to Image Segmentation. The watershed algorithm by means of the Laplacian of Gaussian (LoG) edge detector is making use of to detect the edges of the image and generate the less over-segmented image.

Ghoshale et al. (2013) [7] has explained the several edge sharpening filters and to discover the effect on the output image by means of the watershed algorithm. A spatial sharpening filter and mathematical morphology play a very significant role in the presentation of the segmented images. Morphology is the extraordinary type of filtering and structuring elements used for shape smoothing and elimination of small holes.

Rahman et al. (2013) [8] present, a novel image segmentation method based on an adaptive threshold and masking operation with the watershed algorithm. Whose objective is to overcome an over-segmentation problem of the traditional watershed algorithm

Qinghua Ji, Ronggang Shi [9] article make available the latest method of image segmentation by means of watershed transformation. To make use of morphological opening and closing operations to a method the gradient image aim to reduce the over-segmentation region, and reconstruction of the morphological gradient can maintain the shape of the gradient image. This proposed method can simplify gradient image at the same time as maintaining the outline of the exact location of the dividing line, thereby eradicate the root causes of the phenomenon have been split. The principle of this paper is to discover the early stage of lung cancer and more precise result by using diverse enhancement and segmentation techniques.

Roopali R. Laddha et al. 2016 [10] paper planned a method to detect a brain tumor by means of medical imaging techniques.
Techniques focused in this paper are present Text- Noise Removal \& segmentation which be able to include a technique found on the threshold and watershed segmentation and use the morphological operators. The proposed segmentation process was experimented by means of MRI scanned images of human brains, therefore locating the tumor in the images. Samples of a human brain are there in make use of, a scanned image of MRI process and then were processed through segmentation methods.

\section{PROBLEM DEFINITIONS}

Medical Images are one of the most important concerns of Image processing wherever the Accuracy, Sensitivity, and Specificity are required at extraordinarily high level. From our experimental results, it was noticed that the Region growing Image Segmentation Technique. However, from our experimental results, this research work revealed that this model fails to make efficient to classify intensity causes poor tumor classification of Sensitivity, Specificity, and Accuracy. This is one of the major issues to predict the pattern and to address this issue, the masked-watershed image segmentation Technique is proposed and framework is described in the following Section.

\section{PROPOSED METHODOLOGY}

Now, the method of our projected technique is shown in figure 4.1. The present of an "Over-Segmentation in "watershed segmentation" may be determined by means of the aim of the "Mask-Watershed segmentation" In the preprocessing representation, the de-noised image makes use of to decrease the low-frequency components i.e., to eradicate noise. Secondly, in the post-processing, the masked-watershed segmentation is applied to overcome over-segmentation in watershed segmentation. Then the morphological operation highlights the portion which has a higher intensity than additional regions of the image. Strel is making use of to create a morphological structure of the element. Imerode is used to shrink the image and the filling and expanding the image are done by imdilation. Finally, it is superimposed on the original image and detects the tumor.
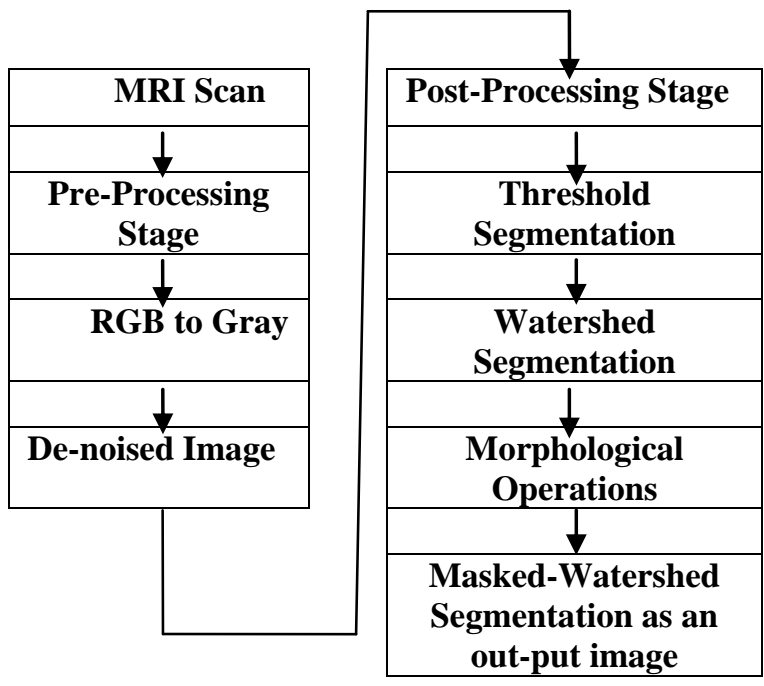

Fig.4.1 The Flow chart of the Proposed Method 


\subsection{MR Image \& Pre-Processing Segmentation}

MR Image is there to be loaded as the input. Function rgb2gray makes use of to convert an image into the gray-scale system. The motive to use this as follows:

A Gray level signifies the interval number of quantization in grayscale image processing. Next, the most commonly make use of storage method is 8-bit storage. There are presently 256 gray levels contained in an 8-bit grayscale image, by means of the side of the similar time the intensity of each pixel, be capable of having from 0 to 255 , with zero being black and 255 being white.

Another commons used storage method is 1-bit storage. Nearby exist two gray levels by means of 0 being black and 1 being white i.e., a binary image, which is generally used in the medical images.

In images, noise is the origin of the random fluctuations in intensity. Noise corresponds to unwanted information which corrupts the image quality. Noise is characterized as a process which has an effect on the acquired image quality with the aim of is being not a part of the original image.

Then image requires a filter for removing the high-intensity component and the above noise. Preprocessing includes the input images of MRI brain tumor and filtering image. In this filtering, the image, several dissimilar filters are able to be used. So, here in this research, Noise is removed by Morphological-based de-noising - Gaussian filtering [11], [12] is efficient and produces better results as compared to other filters. The Gaussian filtering system is supported for the peak detection. The peak detection is based on the reality that peaks are to be desired. The main point in this filter exacts not only the spectral coefficient of interest, although all the amplitude spectrum coefficients surrounded by the filter window. Some properties of Gaussian filter are

1. They reduce edge blurring.

2. They are present linear low pass filters.

3. It is computationally efficient (large filters be present implemented by means of small 1D filters).

4. It executes the same in all directions.

5. The quantity of smoothing is restricted by $\sigma$ (larger $\sigma$ for additional intensive smoothing).

Thus Morphological-based de-noising-Gaussian Filters exist makes use of to attain well-organized results as compared to other filters.

\subsubsection{Post-Segmentation Processing Threshold Techniques}

It is the simplest segmentation method. Here it separates out the ROI which is used for analyzing. This partition is based on the dissimilarity of intensity among the object and the background pixels. Here the comparison of each pixel intensity value by means of respect to a threshold. Previously we have separated accurately the important pixels; we can set them by means of a determined value to identify them (i.e. we can assign them a value of 0 represents (black), 255 represents (white) or any value that goes well with your needs).

Important in the threshold context is the selection of adequate threshold values. According to the way these values are computed, threshold techniques are capable of, this is as shown below:

- Global - threshold values are calculated for the entire image.

- Local - threshold values are calculated for each pixel, based on the information contained within their neighborhood

- Hybrid - threshold values are the result of combining global and local techniques.

\subsubsection{Gradient Magnitude}

The gradient of an image measures how it is altering. It makes available two pieces of information. The magnitude of the gradient informs us how quickly the image is altered while the direction of the gradient tells us the direction in which the image is changing for the most part rapidly. To demonstrate this, think of an image as similar to a landscape, in which at each and every point we exist given a height, somewhat than intensity. For any point in the landscape, the path of the gradient would be the path of the ascending order. The magnitude of the gradient would inform us how quickly our height rises as soon as we take a very small step ascending order. Since the gradient has a direction and a magnitude, it exists natural to encode this information within a vector. The length of this vector presents the magnitude of the gradient whereas its direction gives the gradient direction. Since the gradient may be different in every location, we represent it with a different vector in every image location.

The gradient magnitude is used to pre-process a grayscale image earlier than using the watershed transform for segmentation.

\subsubsection{Morphological Operation}

In some medical image analysis, image segmentation based on the threshold or edge detection is not sufficient because the image feature is deficient or the objects under examination are overlapped. In such applications, morphological segmentation is a successful means of image segmentation. Morphological segmentation partitions an image support on the topographic exterior of the image. It is applied to the improved binary image. The reason of the morphological operator is to separate the tumor part of the image and visible in white color. [11]

Now in Morphological segmentation is a multiple-step procedure involving a number of functions. The following record illustrates each morphological segmentation step by step as given below [11],[12].

Opening: It is a significant morphological operator. It is there classified as erosion, followed by dilation.

Opening by reconstruction: In an image erosion removes pixels from the boundaries of objects. The number of elements to be present, detached from the image under processing is able to be resolved by means of the size and shape of the structural element and restructure the image.

Opening and closing: The morphological closing is used to joint circles in the image and filling the space among them in addition to by means of smoothing their outer edges.

Opening and closing by reconstruction: Dilation adds pixels to the borders of objects in an image. The procedure may be repeated to construct larger effects. By using this image complement of a binary image, zeros be converted into ones and ones be converted into zeros; black and white are reversed. In the complement of an intensity or RGB image 
and the difference is making use of as the pixel value in the output image. Here in this output image, the dark regions become lighter and lighter region become darker.

Opening and closing of regional maxima by reconstruction: Regional maxima are connected components of pixels with an invariable intensity value whose external boundary pixel values are less than the above intensity value. When the pixels are set to one is identified regional maxima and all other pixels are zero. Here the opening and closing followed by reconstruction. Then this image is superimposed on an original image and then modified and removes the connected components. Then followed by Threshold opening-closing by reconstruction, then convert an image into the binary image. Watershed ridge lines are formed.

\subsubsection{Watershed Segmentation}

The Watershed segmentation is used because it is one of the best methods to group pixels of an image lying on the basis of their intensities. Pixel falling under similar intensities is grouped together. It is good segmentation techniques for isolating an image to isolate a tumor from the image. Watershed is a mathematical morphological, function tool. It is normally used for checking output rather than an input segmentation technique because it typically

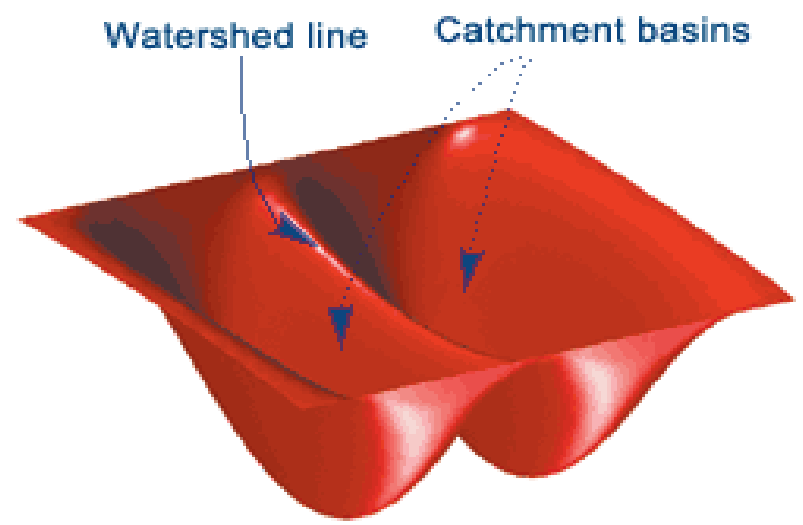

Fig 4.2 Watershed segmentation

Suffers from over segmentation and under the segmentation. It is also suitable for the images that have higher intensity values.

The key word behind using the watershed transform for segmentation is as follows: Change your image into another image whose catchment basins exists the things you desire to identify.

It is classified as a gradient-based segmentation approach. It reflects on the gradient map of the image as a relief map. When the target regions contain small contrast as well as week boundaries, the watershed transformation is capable of offer closed contours. When a topographic relief is flooded with water, the dividing lines in the area of rain falling over the regions structure the watersheds. Naturally, a drop of water falling on a topographic relief flow towards the "nearest" minimum. The purpose of the nearest minimum that lies by the side of the end path of steepest fall. This happens if the point lies within the catchment basin of that minimum.

An approach is to imagine the landscape individual immersed in a lake in which opening (holes) are penetrating in the local minima is called the catchment basin. This is explained as simple as follows. Watershed segmentation is a gradient-based segmentation method. It considers at the same time as the gradient map of the image the same as a relief map. Water filled up by the side of these starting local minima and at points, wherever water coming as of dissimilar basins would meet and dams will be built. When the water levels arrive at the highest peak in the landscape the procedure is stopped up. At the same time, as a result, the landscape is partitioned interested in regions or basins separated by dams, called watershed lines or merely watersheds as shown in the below Figure3.4. i.e., local minima give catchment basin and local maxima give watershed lines

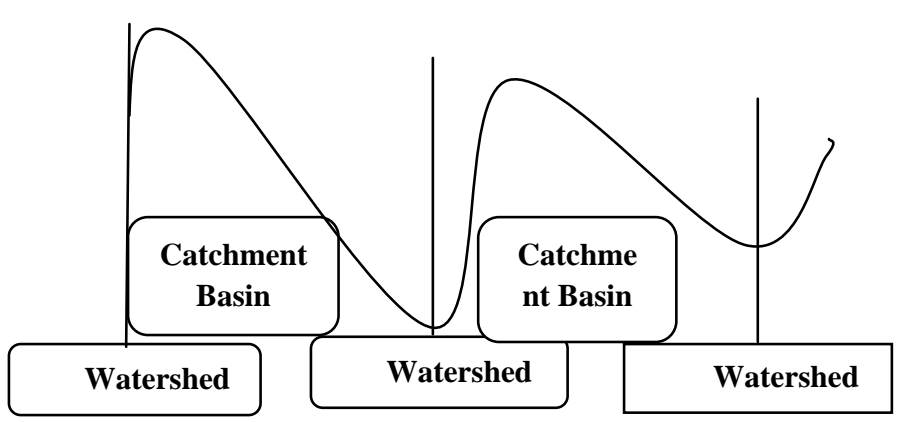

Figure 4.3: Watershed segmentation-local minima yield catchment basins; local maxima define the watershed lines.

The Distance Transform is a familiar tool makes use of in watershed transform for image segmentation. The theory is there the distance from every pixel to its nearest non-zero valued pixel. Every distinctly valued pixel has a distance transform value of 0 , as it is the neighboring non-zero valued pixel of it. An example known under is a binary matrix and its distance transform value.

\begin{tabular}{|l|l|l|l|l|}
\hline $\mathbf{1}$ & $\mathbf{1}$ & $\mathbf{0}$ & $\mathbf{0}$ & $\mathbf{0}$ \\
\hline $\mathbf{1}$ & $\mathbf{1}$ & $\mathbf{0}$ & $\mathbf{0}$ & $\mathbf{0}$ \\
\hline $\mathbf{0}$ & $\mathbf{0}$ & $\mathbf{0}$ & $\mathbf{0}$ & $\mathbf{0}$ \\
\hline $\mathbf{0}$ & $\mathbf{0}$ & $\mathbf{0}$ & $\mathbf{0}$ & $\mathbf{0}$ \\
\hline $\mathbf{0}$ & $\mathbf{1}$ & $\mathbf{1}$ & $\mathbf{1}$ & $\mathbf{0}$ \\
\hline
\end{tabular}

\begin{tabular}{|l|l|l|l|l|}
\hline 0.00 & 0.00 & 1.00 & 2.00 & 3.00 \\
\hline 0.00 & 0.00 & 1.00 & 2.00 & 3.00 \\
\hline 1.00 & 1.00 & 1.41 & 2.00 & 2.24 \\
\hline 1.41 & 1.00 & 1.00 & 1.00 & 1.41 \\
\hline 1.00 & 0.00 & 0.00 & 0.00 & 1.00 \\
\hline
\end{tabular}

\section{Binary Image Matrix}

Distance Transform matrix

Watershed segmentation resolves a variety of image segmentation complexity. It is there suitable for the images that have greater intensity value. Watershed segmentation is end product results over segmentation. Over-segmentation is a segmentation method with specifically fixed parameters is used to acquire an over-segmented end result. Extra information from other segmentation techniques is used to eradicate false boundaries which do not correspond to regions. To control this over segmentation in the watershed segmentation followed by mask watershed segmentation.

\subsubsection{Masked-Watershed Segmentation}

The Mask is used to modify the stored image. This can be done using Boolean logic to get pixels in the grayscale image to the value of the binary image. If it is a non-zero the result of multiplying the gray scale image by the binary image by means of the convention with the aim of the binary image values are 0 for off and 1 for each pixel.

After Masking is completed, then the Morphological Operation tool, it normally used for checking output rather 
than using at the same time as an input segmentation system because it generally suffers from over-segmentation and under segmentation. It is also suitable for the images that have higher intensity values than additional regions of the image. Then reconstruct the image i.e., the image is converted into a binary format. The purpose of this is to separate the tumor portion of the image accurately as shown in white color.

The scope of this study is masked watershed segmentation.

In the field of landscape, the watershed model exist a wellknown tool and the foremost problem by means of the basic watershed transformation is over-segmentation which is reduced in mask watershed segmentation. The techniques are mask watershed segmentation is used to get the reliable results, that is to achieve more Sensitivity, Specificity, and Accuracy segmentation while solving the over segmentation problem at the source of pre-segmentation techniques [2].

\section{RESULT ANALYSIS AND VALIDATION}

This research work has recognized two well-known segmentation technique (I). Region growing and (ii). MaskedWatershed Segmentation. The exhibitions of these two procedures are focused fully by actualizing them and also the execution of Mask-Watershed segmentation is enhanced than the region growing. The ideas and models of region-growing are described as follows

\subsection{Region Growing Algorithm:}

This region growing is a procedure that group's pixels in the whole image into sub-regions. It is processed in four steps

1. Compute a group of seed pixels in an original image.

2. Select a set of similarity criterion, i.e., gray level intensity or color and set up a stopping rule.

3. The growing regions to each seeded are those neighboring pixels that have predefined properties just like that of the seeded pixels.

4. The process Region growing is stopped when no more pixels met the criterion for inclusion in those regions.

The Performance Analysis by region growing is as shown below fig 4.4. a. Input Image, b. RGB to Gray c. Denoising image, d. Segmented Region Growing as an out Put

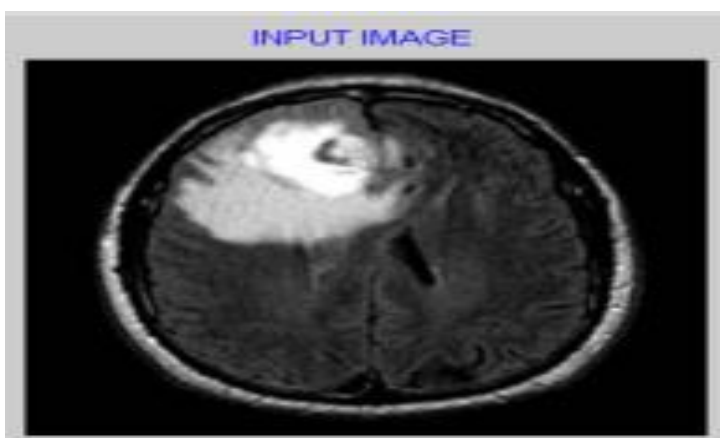

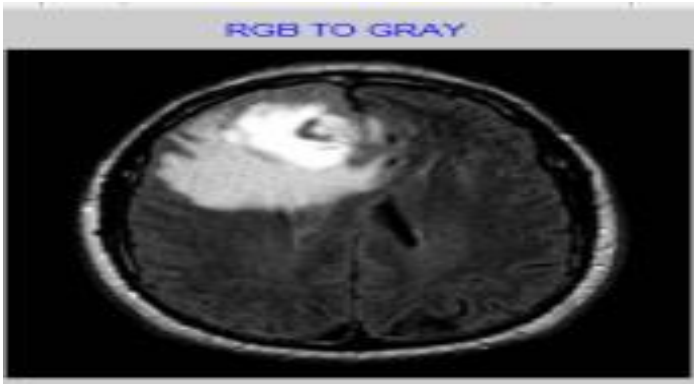
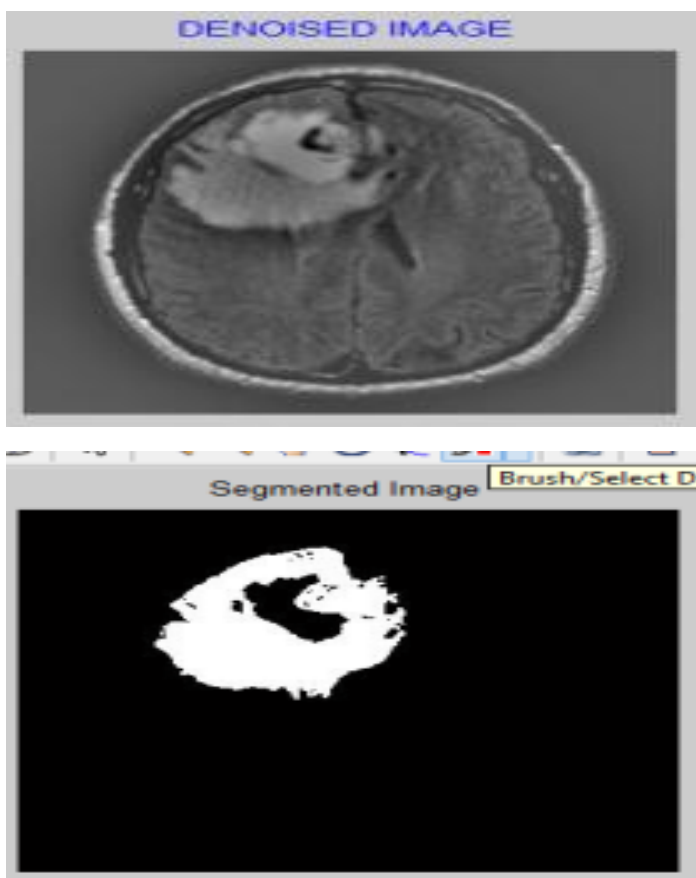

5.2 Accuracy: Accuracy is defined as the condition or the quality of the result is being true i.e., as simple as freedom from error or the defected portion of an object.

5.3 Sensitivity: True positive: In clinic sick people properly recognized as sick

Sensitivity $=$ No of true positive/No of true Positive+ No of False Negative

5.4 Specificity: True negative: Healthy people properly recognized as healthySpecificity=No of True Negative/No of True Negative+ No of False Positive.

In general, Positive $=$ identified and negative $=$ discarded , as a result:

- $\quad$ True positive $=$ properly identified

- True negative = properly rejected

Table5.1: Statistical Measurement of Region Growing

\begin{tabular}{|l|l|l|l|}
\hline Images & Sensitivity & Specificity & Accuracy \\
\hline Img1.jpg & 57.28 & 95.17 & 92.30 \\
\hline Img2.jpg & 28.19 & 98.98 & 96.14 \\
\hline Img3.jpg & 74.42 & 98.11 & 97.60 \\
\hline Img4.jpg & 55.68 & 99.06 & 97.98 \\
\hline Img5.jpg & 17.75 & 99.29 & 98.71 \\
\hline Img6.jpg & 55.43 & 99.29 & 99.28 \\
\hline Img7.jpg & 47.45 & 98.59 & 97.96 \\
\hline
\end{tabular}


From the above Table 5.1. The overall Sensitivity is $48.02 \%$, Specificity is $98.35 \%$, and Accuracy is $96.99 \%$ is found for Region Growing Segmentation techniques

The performance of the proposed method can be evaluated by using Mat lab R2013a and 8.1 versions. Let us take one example in which we have one medical image Img1.jpg, the RGB medical image, which is converted into the normalized format by using de-noising to obtain a filtered image, then converted into, and then high-level segmentation is performed, then low- level segmentation to extract the accurate infected region from the whole medical image. The experimental results of Masked-Watershed Segmentation are as follows in the following 7steps.

\section{Step 1: Input MR Images}

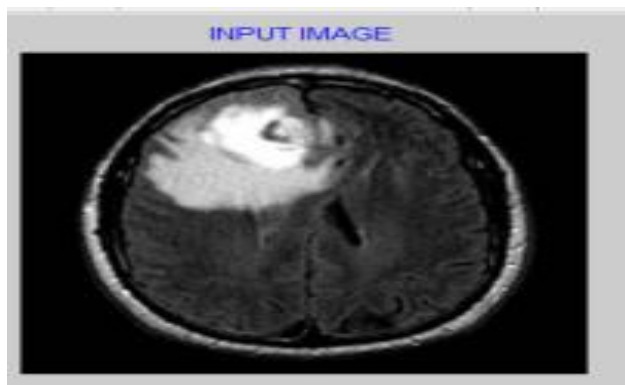

Step 2: RGB to Gray

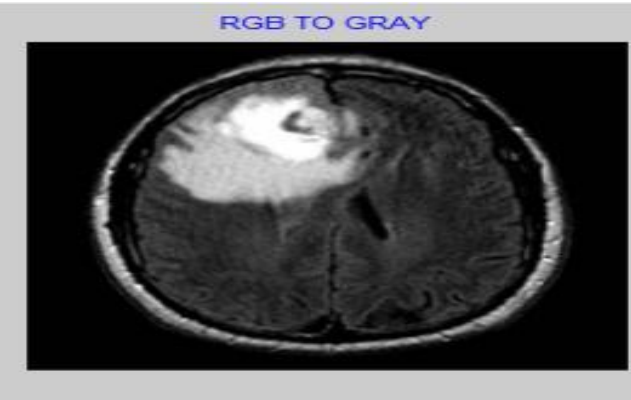

Step3: De-Noised Image

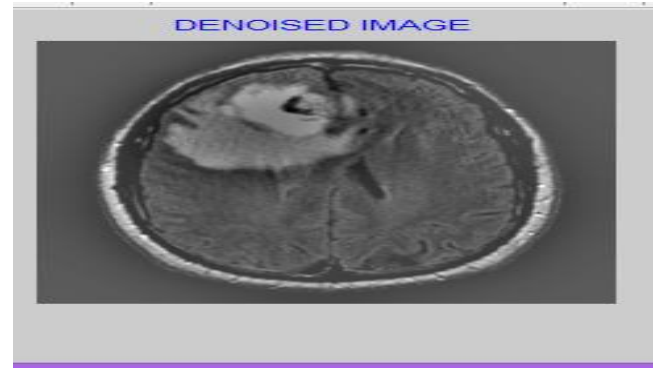

Step 4 Watershed Transform of Gradient Mag.

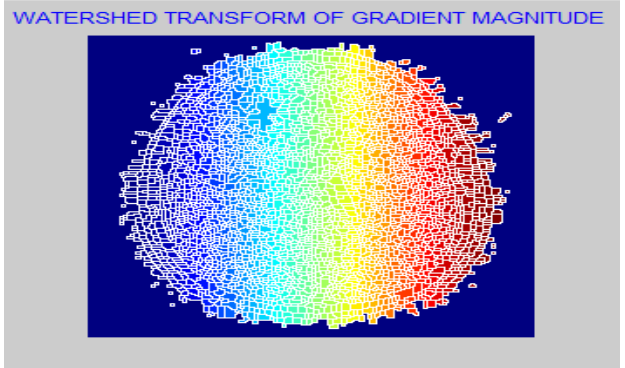

Step 5 Watershed Ridge Lines

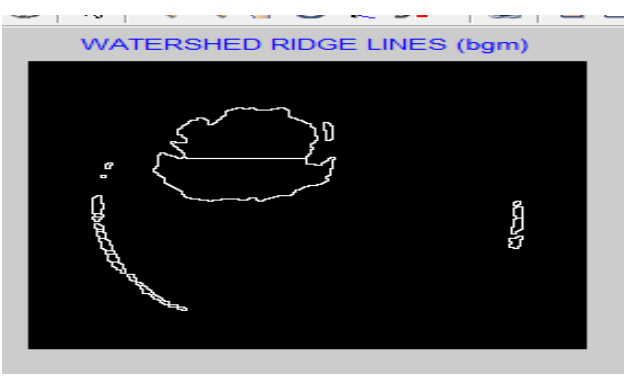

Step 6 Watershed Segmentation

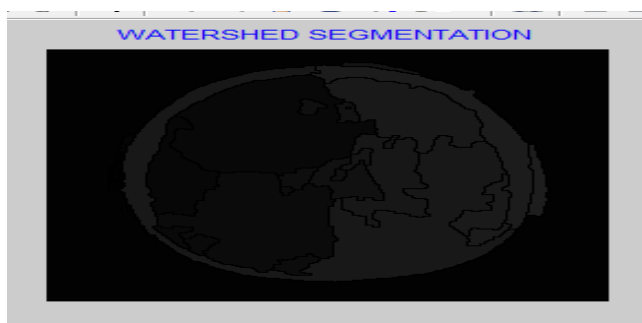

Step 7: Masked-Watershed Segmentation
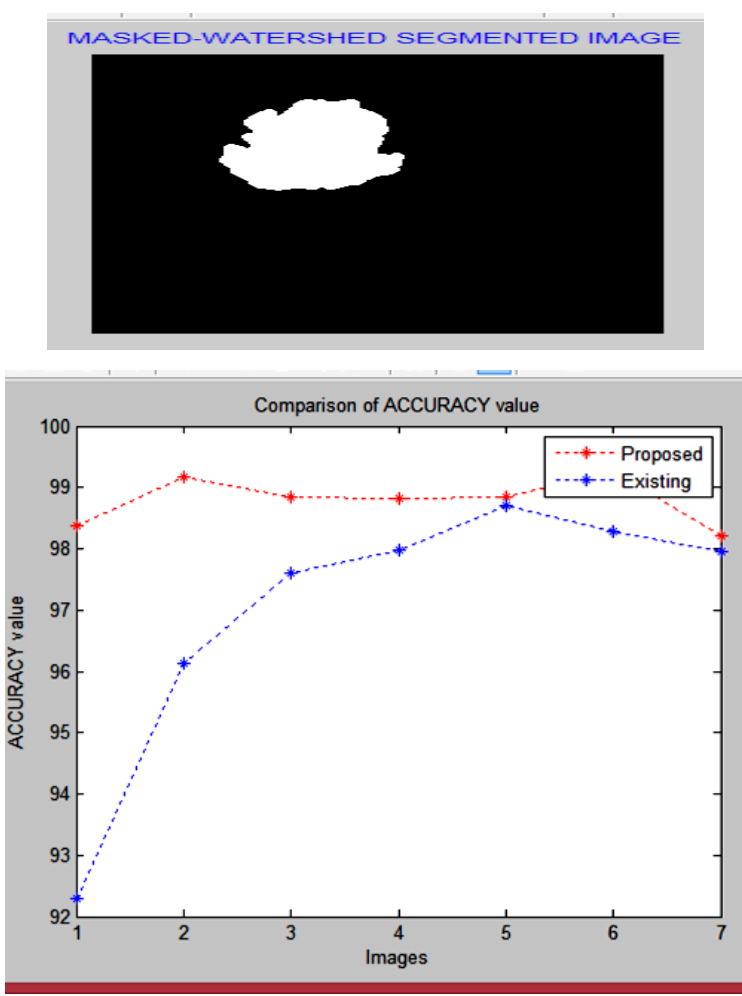

Figure5.1 Accuracy(Region Growing vs MaskedWatershed segmentation) 


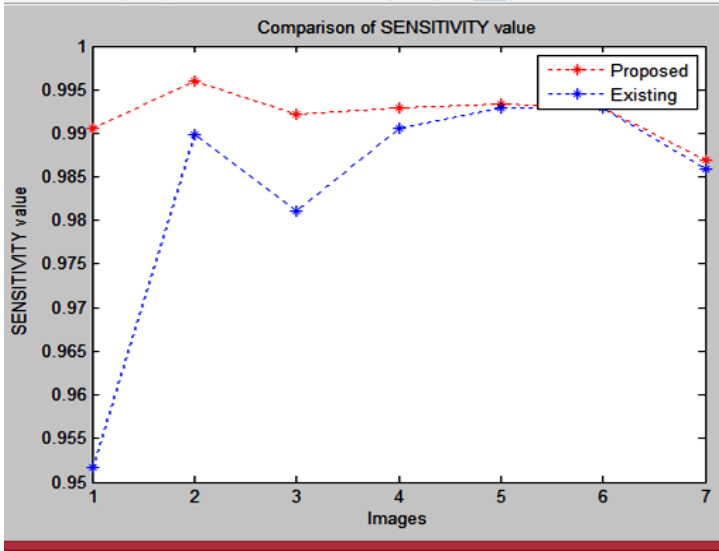

Figure.5.2Sensitivity(Region Growing vs MaskedWatershed)

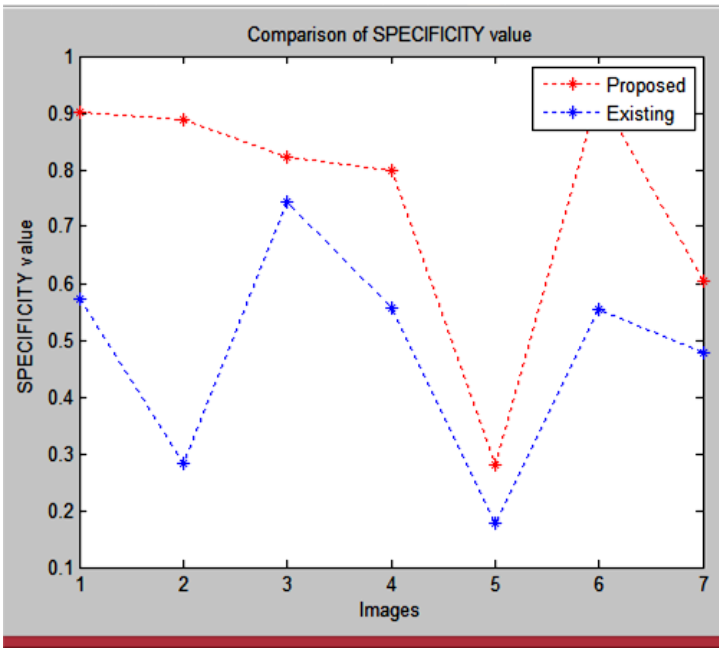

Fig5.3.Specificity(Region Growing vs Masked-Watershed)

Table5.2: Statistical Measurement of Masked-Watershed Segmentation

\begin{tabular}{|l|l|l|l|}
\hline Images & Sensitivity & Specificity & Accuracy \\
\hline Img1.igp & 90.11 & 99.05 & 98.37 \\
\hline Img2.igp & 88.83 & 99.60 & 99.17 \\
\hline Img3.igp & 82.26 & 99.22 & 98.85 \\
\hline Img4.igp & 79.86 & 99.29 & 98.81 \\
\hline Img5.igp & 27.92 & 99.34 & 98.84 \\
\hline Img6.igp & 93.55 & 99.29 & 99.26 \\
\hline Img7.igp & 60.27 & 98.69 & 98.21 \\
\hline
\end{tabular}

From the table 5.2 represented the overall Sensitivity is $74.68 \%$, Specificity is $99.21 \%$,Accuracy is $98.78 \%$ is discovered for Masked-Watershed Segmentation Technique.

\section{CONCLUSIONS}

This research work has proposed Masked-Watershed Segmentation Technique and implemented with MATLAB Tool. The efficiency of the proposed technique is compared with that of existing Region Growing Segmentation Technique and established that the proposed model is performing well in terms of Sensitivity, Specificity, and Accuracy. In future design an image representation of automatic detection of Masked-watershed segmentation of some other medical images.

\section{REFERENCES}

[1] Changmin Zhang, Shuaiqi Zhang, Junxia $\mathrm{Wu}$ and Shaoxiong Han, "An improved wa- tershed algorithm for color image segmentation," 2012 International Conference on Computer Science and Electronics Engineering (ICCSEE), Year 2012, pp.69-72.

[2] Md. Sharifur Rahman and Md. Rafiqual Islam, "Counting objects in an image by marker controlled watershed segmentation and thresholding," 3rd IEEE International on Advance Computing Conference (IACC), Year 2013, pp.1251-1256.

[3] Xianwei Han, Yili Fu and Haifeng Zhang, "A fast twostep marker-controlled watershed image segmentation method, "International Conference on Mechatronics and Automa- tion (ICMA), Year 2012, pp.1375-1380. ||

[4] Boren Li, Mao Pan, and Zixing Wu, "An improved segmentation of high spatial res- olution remote sensing image using Marker-based Watershed Algorithm," 20th IEEE International Conference onGeoinformatics , Year 2012, pp.1-5. ||

[5] Farheen K. Siddiqui and Vineet Richhariya,"An Efficient Image Segmentation Approach through Enhanced Watershed Algorithm," Computer Engineering and Intelligent Systems, Vol.4,No.6, pp 1-7,2013. ||

[6] P.P. Acharjya, A. Sinha, S. Sarkar, S. Dey and S. Ghosh,"A New Approach Of Watershed Algorithm Using Distance Transform Applied To Image Segmentation,"International Journal of Innovative Research in Computer and Communication Engineering, Vol.1,No.2, pp 185-189, April 2013. ||

[7] Dibyendu Ghoshal and Pinaki Pratim Acharjya,"Effect of Various Spatial Sharpening Filters on the Performance of the Segmented Images using Watershed Approach based on Image Gradient Magnitude and Direction," International Journal of Computer Applications, Vol. 82, No.6, pp 19-25, November 2013. ||

[8] Md.Habibur Rahman and Md.Rafiqual Islam, "Segmentation of color image using adaptive thresholding and masking with watershed algorithm," IEEE International Conference on Informatics, Electronics \& Vision (ICIEV), Year 2013, pp.1-6. ||

[9] Rabul H Laskar, Kalyan Banerjee and Debajit Basak, "Removal of High Density Salt and Pepper Noise from Color Images through Variable Window Size," IEEE International Conference on Circuits, Power and Computing Technologies, Year 2013, pp. 1132-1136. ||

[10] M.Sonka, V.Hlavac, and R.Boyle, Image Processing, Analysis, and Machine Vision, PWS Publishing, 1999. shed transform method provides better segmentation result without preprocessing. Zhang et al. (2012)

[11] P. Perona, J. Malik. "Scale space and edge detection using anisotropic diffusion." IEEE Transaction Pattern Analysis Machine Intell.12 629-639 (1990).

[12] W. Chen, M. Ding, Y. Miao, L. Luo, "Ultrasound image denoising with multi-shape patches aggregation based non-local means," IEEE ICBMI, pp. 14-17, Dec. (2011). [12] W. Chen, M. Ding, Y. Miao, L. Luo, “ 